\title{
A planet around the evolved intermediate-mass star HD $110014^{\star, \star \star}$
}

\author{
J. R. de Medeiros ${ }^{1}$, J. Setiawan ${ }^{2}$, A. P. Hatzes ${ }^{3}$, L. Pasquini ${ }^{4}$, L. Girardi ${ }^{5}$, S. Udry ${ }^{6}$, M. P. Döllinger ${ }^{4}$, and L. da Silva ${ }^{7}$ \\ ${ }^{1}$ Departamento de Física, Universidade Federal do Rio Grande do Norte, Natal, Brazil \\ e-mail: renan@dfte.ufrn.br \\ 2 Max-Planck-Institut fur Astronomie, Königstuhl 17, 69117 Heidelberg, Germany \\ 3 Thueringer Landessternwarte, Tautenburg, Germany \\ 4 European Southern Observatory, Garching bei Munchen, Germany \\ 5 Osservatorio Astronomico di Padova-INAF, Padova, Italy \\ ${ }^{6}$ Geneva Observatory, Geneva University, Geneva, Switzerland \\ 7 Observatório Nacional, Rio de Janeiro, Brazil
}

Received 13 January 2009 / Accepted 10 July 2009

\section{ABSTRACT}

Context. We found evidence for a sub-stellar companion around the K giant star HD 110014. This cool evolved star, with a spectral type K2III and an estimated mass between 1.9 and $2.4 M_{\odot}$, is slightly metal rich with $[\mathrm{Fe} / \mathrm{H}]=0.19$ and a rotational velocity $V \sin i=2.0 \mathrm{~km} \mathrm{~s}^{-1}$.

Aims. To search for extrasolar planets around intermediate-mass stars and to improve our knowledge of the nature of radial velocity variations shown by $\mathrm{G}$ and $\mathrm{K}$ giant stars.

Methods. Based on radial velocity analysis, we found evidence for a substellar companion with a planetary mass and long orbital period. The Radial velocity variation of HD 110014 has been monitored from 2000 until 2007 with FEROS at $1.5 \mathrm{~m}$ ESO and at the $2.2 \mathrm{~m} \mathrm{MPG/ESO,} \mathrm{HARPS} \mathrm{at} \mathrm{the} 3.6 \mathrm{~m}$ ESO and Coralie at $1.2 \mathrm{~m}$ Leonard Euler swiss telescopes in La Silla observatory. The radial velocities were computed by using a cross-correlation technique. Line bisector, Hipparcos photometry and chromospheric lines were analyzed to exclude other root-causes for the radial velocity variability.

Results. We report the presence of an extrasolar planet around the giant star HD 110014, with an orbital period of 835.48 \pm 6.04 days. A Keplerian orbit, with an eccentricity $e=0.462 \pm 0.069$, yields a minimum mass $M \sin i=11.09 M_{\text {Jup }}$. The analysis of the residuals shows evidence for a second RV variability with a period of 130 days and an amplitude of $\pm 100 \mathrm{~ms}^{-1}$. Its nature is not completely clear, but a second planet is a possible explanation.

Key words. stars: evolution - technique: radial velocities - stars: planetary systems - stars late-type

\section{Introduction}

Since the pioneering discovery by Mayor \& Queloz (1995), a little more than a decade ago, of a planet orbiting the star 51 Pegasi, there is now a growing list of surveys searching for extrasolar planets around stars, including objects which are in other evolutionary stages than the main-sequence (MS). In particular, the literature reports today several surveys by radial velocity searching for planets around evolved stars (Frink et al. 2002; Hatzes et al. 2003, 2005; Setiawan et al. 2003, 2005; Sato et al. 2003, 2007; Reffert et al. 2006; Johnson et al. 2007; Niedzielski et al. 2007; Döllinger et al. 2007; Lovis \& Mayor 2007). Indeed, the study of planets around cool evolved stars is a very exciting topic, because it brings new insights to planet formation theories by virtue of the broader stellar mass and age range spanned by the parent stars. This in contrast to the dwarf solar-type stars that have been the subject of most planet searches surveys carried out up to date. In addition, a statistically robust sample of

\footnotetext{
* Based on observations collected at the 1.52-m ESO telescope (1999 to 2002), at the 2.2-m MPG/ESO telescope (2003 to 2007), at the 3.60 ESO telescope and at the Euler swiss telescope, at the La Silla Observatory, Chile.

$\star \star$ Radial velocity measurements are only available in electronic form at the CDS via anonymous ftp to

cdsarc.u-strasbg.fr $(130.79 .128 .5)$ or via

http://cdsweb.u-strasbg.fr/cgi-bin/qcat? J/A+A/504/617
}

extrasolar planets around evolved stars will offer an experimental basis to the study of the dynamics of planetary systems orbiting evolving stars (e.g.: Duncan \& Lissauer 1998), as well as to the problem of a long-term survival of planetary systems along the latest stages of the stellar evolution. So far about 20 substellar companions orbiting intermediate-mass evolved stars have been detected, all by radial velocity surveys. Despite the still limited number of detections, these extrasolar planets point to different trends with respect to those discovered around mainsequence stars. For instance, Pasquini et al. (2007) have shown that the metallicity distribution of giant stars hosting planets is very different from that of planet-hosting main sequence stars. In contrast with the trend observed for dwarf solar-type stars with Jupiter-size planets, giants hosting planets do not favor high metallicity objects, rather they follow the same age-metallicity distribution of all the stars surveyed. In addition, a trend for high frequency of massive planets (Lovis \& Mayor 2007) and the lack of inner planets (Jonhson et al. 2007) are other characteristics observed among planets around evolved, intermediate-mass giant stars.

In this paper we report the discovery of a substellar companion around the intermediate-mass giant star HD 110014, based on observations from three spectrographs. Great care was taken in the analysis to evaluate the presence of intrinsic stellar phenomena that can also produce variations, mimicking the gravitational influence of planetary mass companions. Rotational 
Table 1. Stellar parameters of HD 110014.

\begin{tabular}{lll}
\hline \hline Spectral type & K2III & \\
$V$ & 4.66 & $\mathrm{mag}$ \\
$M_{v}$ & $-0.11 \pm 0.06$ & $\mathrm{mag}$ \\
$(B-V)$ & 1.24 & $\mathrm{mag}$ \\
Parallax & $11.11 \pm 0.29$ & $\mathrm{mas}$ \\
Distance & $90.0 \pm 2.3$ & $\mathrm{pc}$ \\
$T_{\text {eff }}$ & $4445 \pm 70$ & $\mathrm{~K}$ \\
{$[\mathrm{Fe} / \mathrm{H}]$} & $0.19 \pm 0.05$ & \\
Age & $0.95 \pm 0.40$ & $\mathrm{Gyr}$ \\
$M$ & $2.17 \pm 0.28$ & $M_{\odot}$ \\
Log $g$ & $2.10 \pm 0.10$ & $\mathrm{c.g} . \mathrm{s}$. \\
$R$ & $20.9 \pm 1.1$ & $R_{\odot}$ \\
$E(b-v)$ & $0.05 \pm 0.04$ & $\mathrm{mag}$ \\
$\theta$ & $2.16 \pm 0.13$ & $\mathrm{mas}$ \\
$V \sin i$ & 2.0 & $\mathrm{~km} \mathrm{~s}{ }^{-1}$ \\
$P_{\text {rot }} / \sin i$ & 513 & day \\
\hline
\end{tabular}

The stellar parameters were originally computed by da Silva et al. (2006). $\log (g)$, Mass, Radius and age have been re-computed in the present paper after adopting the new Hipparcos parallaxes (van Leeuwen 2007).

modulation and activity can be excluded as the source of the radial velocity variations, as described in the following sections.

\section{The giant star HD 110014}

HD 110014 has an apparent magnitude of $V=4.66$ and is classified in SIMBAD as a K2III giant star. The astrometric parallax from the Hipparcos catalogue is $\pi=10.24 \pm 0.88$ mas (ESA 1997) and $11.11 \pm 0.29$ mas in the Hipparcos new reduction from van Leeuwen (2007). The new parallax is close to the upper limit of the previous value, and its uncertainty is reduced by a factor of about 3 . Since the arguments for preferring the new parallaxes are very compelling (see van Leeuwen \& Fantino 2005; van Leeuwen 2008), the new value is used in this paper. In this case, the distance, distance modulus and absolute magnitude of HD 110014 are, respectively, $d=90.0 \pm 2.3 \mathrm{pc}$, $(m-M)_{0}=4.77 \pm 0.06 \mathrm{mag}$, and $M_{V}=-0.11 \pm 0.06 \mathrm{mag}$. According the Hipparcos catalogue the colour index for this star is $(B-V)=1.24$.

Using the appropriate bolometric correction (Flower 1996) and the solar absolute magnitude of 4.746 (Lejeune et al. 1998), we obtain a luminosity $L=154 L_{\odot}$. The stellar parameters of HD 110014 are listed in Table 1. The values of the parameters, $T_{\text {eff }}[\mathrm{Fe} / \mathrm{H}], \log (g)$ and the stellar radius $R$, as derived from the observed spectra, were computed by da Silva et al. (2006), via their detailed PDF analysis. Errors in $T_{\text {eff }}$ are $70 \mathrm{~K}$ and in $[\mathrm{Fe} / \mathrm{H}]$ 0.05 dex. $E(b-v)$ stands for the observed $(B-V)$ minus the estimated intrinsic $(B-V)_{0}$ (da Silva et al. 2006). Except for the stellar parameters listed by da Silva et al. (2006), no previous investigation has derived stellar parameters for HD 110014. Because of the new parallax value, the values reported in Table 1 have been re-computed in the present paper (cf. Sect. 3.3), but they do not differ substantially from those obtained by da Silva et al. (2006).

Using the CORAVEL cross-correlation peak we have computed a projected rotational velocity $V \sin i<2.0 \mathrm{~km} \mathrm{~s}^{-1}$, by applying the cross-correlation method (Benz \& Mayor 1984; de Medeiros \& Mayor 1999). The same value $\left(<2.0 \mathrm{~km} \mathrm{~s}^{-1}\right)$ is obtained by using the FEROS spectra and the calibration of Melo et al. (2001). This value is typical of the $V \sin i$ value for
K2III giants (de Medeiros et al. 1996). From the projected rotational velocity $V \sin i$ and the adopted stellar radius, listed in Table 1, we have estimated the upper limit of the rotation period as $P_{\text {rot }} / \sin i \sim 530$ days.

\subsection{Mass and age estimates}

The mass of HD 110014 can be estimated by means of its position in the colour-magnitude diagram. The idea is that for single evolved stars this position is strongly (but not uniquely) determined by the stellar mass and metallicity. There is also a very tight inverse relationship between the mass and the stellar age, determined by the power-like relation between the turn-off mass and the main sequence lifetime.

One method that explores this idea has been described in da Silva et al. (2006). It is a slightly modified version of the "Bayesian estimation method" of Jørgensen \& Lindegren (2005). In brief, the method involves the summation of the relative probabilities that a given observed star has been drawn from every small section of all possible isochrones (from Girardi et al. 2002) that encompass the range of observed absolute magnitude, $T_{\text {eff }}$, and metallicity. The method assumes a reasonable age distribution function for the stars in the sample, and returns the probability distribution function (PDF) of variables such as mass, age, radius and surface gravity. Although the method relies highly on theoretical evolutionary tracks and isochrones and in particular on the position of their red giant branches - for giants in the Hyades open cluster it has produced ages in agreement with the main sequence turn-off age. It has also produced a consistent age for the open cluster IC 4651 (Biazzo et al. 2007) and it has provided independent estimates of stellar diameters, in close agreement with those derived from different observational methods (see da Silva et al. 2006).

We apply this method to HD 110014, obtaining the PDFs depicted in Fig. 1. The straight mean and standard errors for the age, mass, surface gravity, radius, and intrinsic colour are, respectively, $t=0.95 \pm 0.40 \mathrm{Gyr}, M=2.17 \pm 0.28 M_{\odot}$, $\log g$ (c.g.s. $)=2.10 \pm 0.11, R=20.9 \pm 1.6 R_{\odot}$ and $(B-V)_{0}=$ $1.25 \pm 0.05$. However, Fig. 1 clearly shows double-peaked PDFs for the age and mass. The double peaks arise from the properties of HD $110014\left(M_{V}, T_{\text {eff }}\right.$, and $\left.[\mathrm{Fe} / \mathrm{H}]\right)$ corresponding, with comparable probabilities, to two very different evolutionary configurations: HD 110014 could be either a $1.9 M_{\odot}$ star $(1.55-\mathrm{Gyr}$ old) at the end of its core helium burning phase, or a $2.4 M_{\odot}$ star (0.68-Gyr old) in its first-ascent RGB, close to the core helium flash. These two configurations are illustrated by the isochrones in the top-left panel of Fig. 1. Discriminating between these two cases is quite difficult at this stage. These two kinds of stars might have different surface chemical compositions, but these differences are expected to be very modest. Tomkin et al. (1976) have measured for this star a rather high ${ }^{12} \mathrm{C} /{ }^{13} \mathrm{C}$ ratio of 28 . Following the most recent work on the subject, such a high ratio is only found in giants with relatively high mass, above $2 M_{\odot}$ (Smiljanic et al. 2008). Given the range of masses we are exploring, this result is very marginal for our star, but it would favor the higher mass solution for HD 110014.

Given this situation, we shall better refer to the confidence levels for the stellar mass as being:

- with $95 \%$ probability in the interval between 1.68 and $2.69 M_{\odot}$;

- with $67 \%$ probability in the interval between 1.86 and $2.47 M_{\odot}$. 

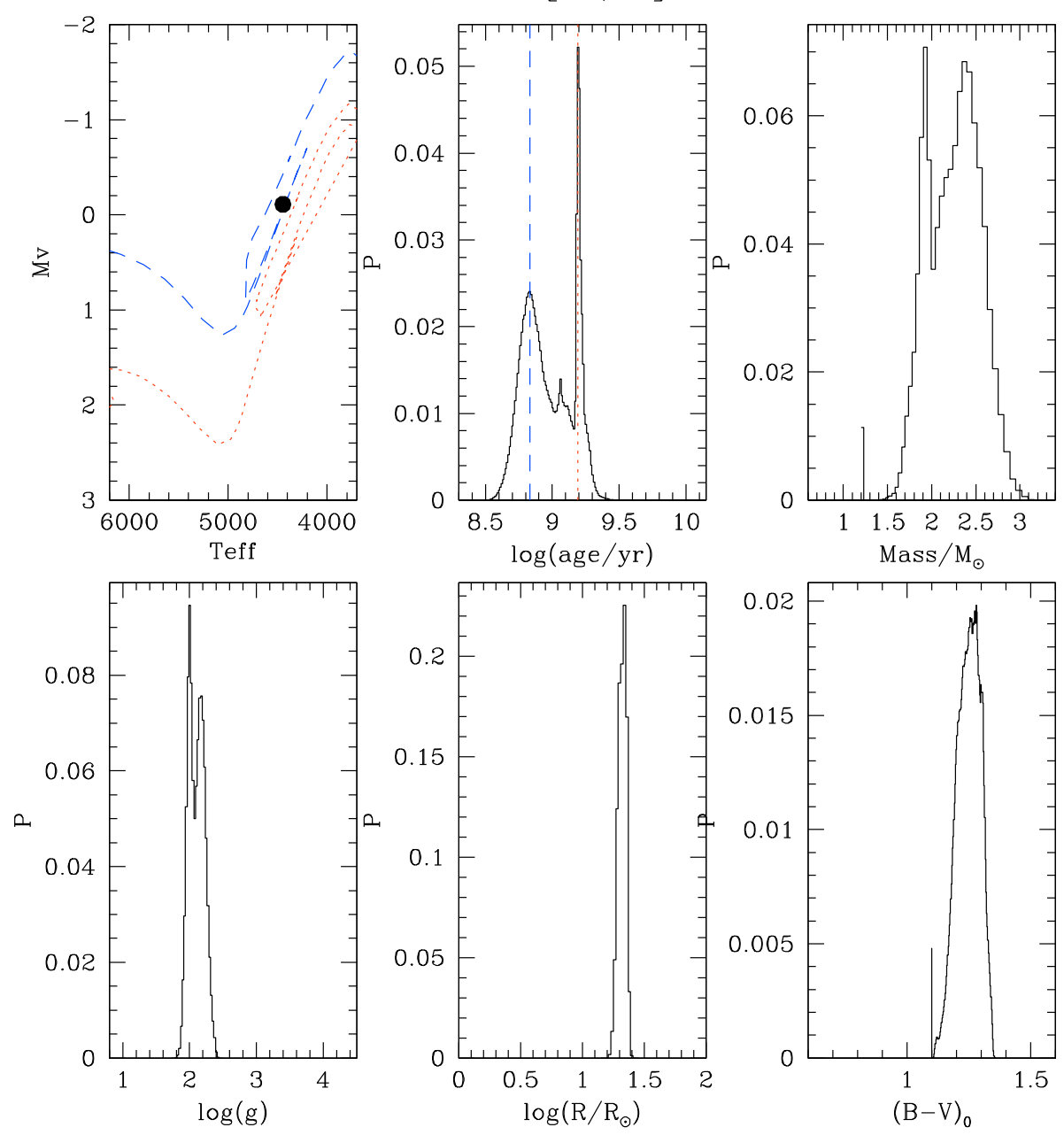

Fig. 1. Results of the determination of stellar parameters via the PDF method. The top left panel shows the position of HD 110014 in the $M_{V}$ versus $T_{\text {eff }}$ diagram (dot), together with two of the $[\mathrm{Fe} / \mathrm{H}]=+0.19$ isochrones that best fit its position - namely the 0.68 and $1.55 \mathrm{Gyr}$ ones (dashed and dotted lines, respectively). In the top middle panel, the same isochrones are marked along the full PDF for the stellar ages. The other panels show, in sequence, the PDFs for the stellar mass, surface gravity, radius, and $(B-V)_{0}$.

These confidence intervals reflect the standard errors in the parallax, photometry, $T_{\text {eff }}$, and $[\mathrm{Fe} / \mathrm{H}]$, but do not consider the possible systematic errors associated with the evolutionary tracks and isochrones in use (from Girardi et al. 2002), and the adopted Bayesian priors (for instance, the method assumes an even distribution of all stellar ages, which might not be the best option). Reducing systematic errors would require a substantial work of fine-tuning the PDF method and the evolutionary tracks of giant stars, with the aid of stars in clusters and binaries, which is far beyond the scope of this paper. For the moment, it is enough to recall that the PDF method has put HD 110014 on the same mass/age scale of that of the sample by da Silva et al. (2006), which is reasonably well calibrated for the Hyades giants.

\section{Observations and data analysis}

We have monitored the radial velocity of HD 110014 in the scope of our program to search for extrasolar planets around giant stars that was started in October 1999 (Setiawan et al. 2003, 2004). Up to October 2008, we have collected measurements at 49 epochs with three echelle spectrographs as follow: (i) 32 measurements with FEROS $(R=48000)$, first at the $1.52 \mathrm{~m}$ ESO (1999 to 2002), and latter at the 2.2 m MPG/ESO (2003 to 2007) during the MPG guarantee time, telescopes; (ii) 10 measurements with HARPS $(R=120000)$ at the $3.60 \mathrm{~m}$ ESO telescope; and (iii) 7 measurements with CORALIE $(R=50000)$, at the Euler swiss telescope. The details of the instrumental setup and data reduction of the FEROS measurements can be found in Setiawan et al. (2003), whereas the data acquired by HARPS and CORALIE were reduced by their authomatic online Data Reduction Softwares (DRS) (Baranne et al. 1996).

Given the use of three different instruments and data analysis software, zero point shifts may be present among the different data points, and thus systematic effects need to be evaluated. We recall for instance that the observed spectra are cross-correlated with numerical masks, optimized for stellar spectral type and luminosity. For the FEROS (both $2.2 \mathrm{~m}$ and $1.5 \mathrm{~m}$ ) and CORALIE spectra, the same mask (K0) has been used, and no major shifts are expected. This hypothesis is well confirmed experimentally (see below). As far as HARPS is concerned, the spectra were processed with a K5 mask, and an offset is expected. By comparing FEROS and HARPS spectra of $\mathrm{K}$ giants taken a few days apart, an offset of about $180 \mathrm{~ms}^{-1}$ (F-H) is expected.

The time series of the radial velocity measurements for HD 110014, displayed in Fig. 2, show a clear long-period variability. A Lomb-Scargle periodogram analysis (Scargle 1982) yields significant power at a period of 835 days (Fig. 3). The false alarm probability (FAP) of this peak, using expressions given in Scargle (1982) is estimated to be $10^{-8}$.

Assuming the periodic radial velocity variation is due to an orbiting companion, we fitted a Keplerian orbit for HD 110014 , obtaining an orbital period of $835.477 \pm 6.04$ day, an eccentricity $e=0.462 \pm 0.069$, with a radial velocity semi-amplitude $K=$ $158.2 \pm 14.4 \mathrm{~ms}^{-1}$, leading to a minimum mass $M \sin i=11.1$ 

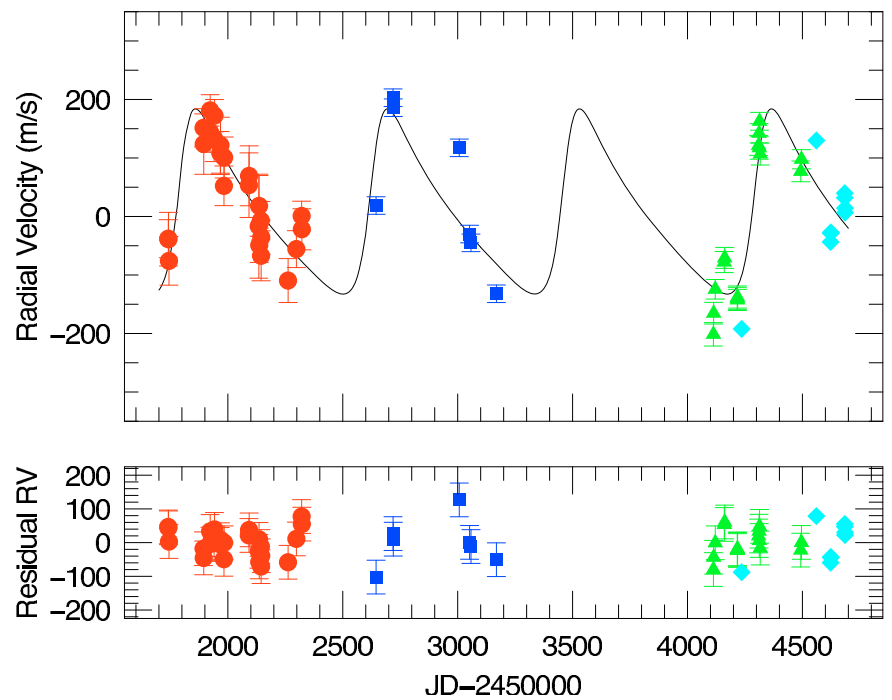

Fig. 2. Radial velocity measurements and the best fit orbit (solid line) for HD 110014 (upper panel) and the residual radial velocity variations, after subtracting the contribution of the planet orbit (lower panel). Red points: FEROS at $1.5 \mathrm{~m}$ telescope, Light Blue points: FEROS at 2.2.m telescope; Green points: HARPS; Blue points: Coralie.

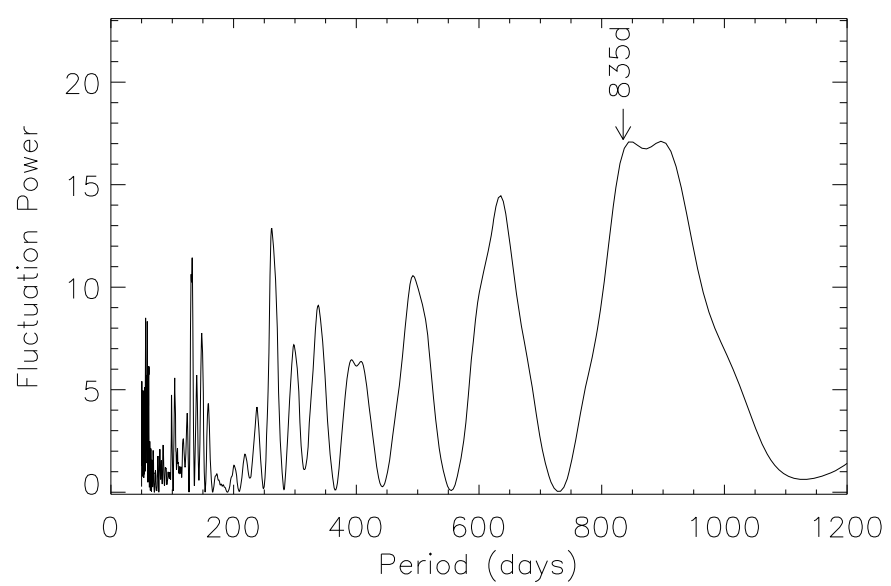

Fig. 3. The LombScargle periodogram of the radial velocity measurements, with a significant power at a period of 835 days.

(9.5) $M_{\mathrm{Jup}}$ and a semi-major axis $a=2.33$ (2.15) AU, where the number within parenthesis refer to the choice of a stellar mass $2.4 M_{\odot}$ and the one without parenthesis for a choice of $1.9 M_{\odot}$. These orbital parameters are listed in Table 2, whereas Fig. 4 shows the radial velocity variations phase-folded to the orbital period.

We note here that in our procedure the instrumental zero point shifts for each data set were left as a free parameter, to be fitted by the minimization procedure. The zero point shifts have been found, as expected, to be very small: -8 and $-5 \mathrm{~ms}^{-1}$ between FEROS $1.5 \mathrm{~m}$ - FEROS $2.2 \mathrm{~m}$ and FEROS $15 \mathrm{~m}-$ Coralie respectively. As far as HARPS is concerned, a shift of $-220 \mathrm{~ms}^{-1}$ with respect to FEROS $(1.5 \mathrm{~m})$ has been computed, slightly larger (about $40 \mathrm{~ms}^{-1}$ ) than what found from the direct comparison between the other stars. The origin of this discrepancy is not clear and studies are now in progress to better settle this matter. Nevertheless, given the relatively small number of the HARPS points, the discrepancy in the offset does not influence our results. This discrepancy is probably not a concern considering that $\mathrm{K}$ giants can exhibit significant intrinsic variability due to stellar oscillations (see below). The relative offsets

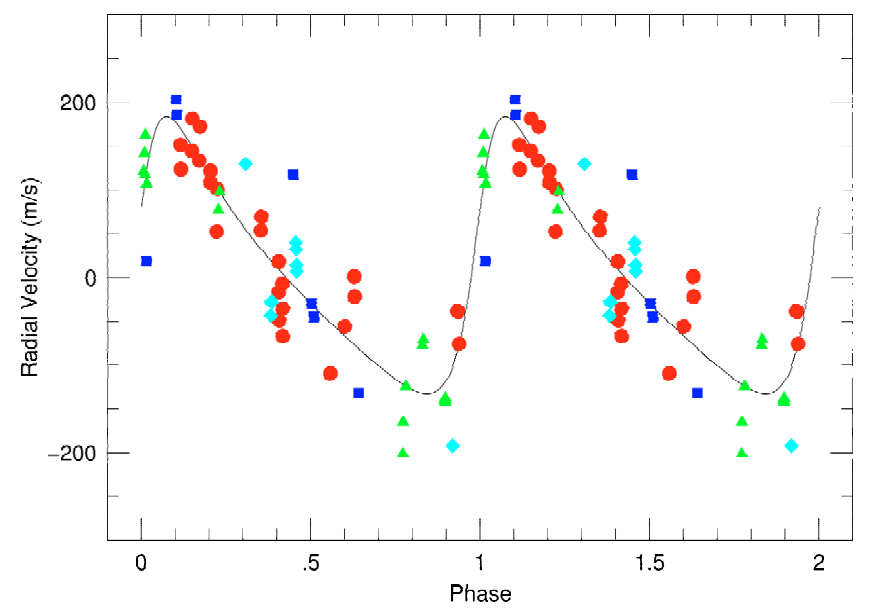

Fig. 4. Radial velocity measurements of HD 110014, phased to the orbital period of 835.477 days. The solid line represents the Keplerian orbital solution.

Table 2. Orbital parameters for HD 110014 b. Mass of the planet $M_{2}$ and semi axis $a_{1}$ are given for a stellar mass of 1.9 and $2.4 M_{\odot}$ separately.

\begin{tabular}{lll}
\hline \hline$P$ & $835.477 \pm 6.04$ & day \\
$T_{0}(\mathrm{JD})$ & $2451796.9065 \pm 5.1$ & day \\
$e$ & $0.462 \pm 0.069$ & \\
$V$ & $-18.62 \pm 0.12$ & $\mathrm{~km} \mathrm{~s}^{-1}$ \\
$\omega_{1}$ & $290.6 \pm 3.8$ & $\mathrm{deg}$ \\
$K_{1}$ & $158.2 \pm 14.4$ & $\mathrm{~ms}^{-1}$ \\
$\sigma(\mathrm{O}-\mathrm{C})$ & 45.8 & $\mathrm{~ms}^{-1}$ \\
$f(\mathrm{~m})$ & $(2.37 \pm 0.64) \times 10^{-7} M_{\odot}$ & \\
$a$ & $2.14(2.32) \pm 0.03$ & $\mathrm{AU}$ \\
$m_{2} \sin i$ & $11.09(9.5) \pm 1$ & $M_{\text {Jup }}$ \\
\hline
\end{tabular}

between RV data from the various spectrographs were calculated following a least squares procedure in the orbit fitting. Relatively few RV measurements from an instrument coupled with large intrinsic variability means that the relative RV offset may not be accurately determined. Furthermore, the error of the offset determination for each spectrograph is $\approx 15 \mathrm{~ms}^{-1}$ so that the relative offset between two spectrographs can be $\approx 20 \mathrm{~ms}^{-1}$.

Although the orbital fit to our radial velocity measurements is very good there are points significantly deviating from the obtained solution, yielding a rms scatter of about or $45.8 \mathrm{~ms}^{-1}$, with a $\chi^{2}$ of 0.90 . In the lower panel of Fig. 2, that shows the residual radial velocity variations, after removal of the orbital contribution due to the planetary companion, one observes a clear variability, with a period much shorter than the orbital period of 835.477 days. Indeed, a Lomb-Scargle periodogram analysis of these radial velocity residuals shows a peak at a period of 130 days (Fig. 5). When a hypothetical Keplerian orbit is subtracted with a period of 130 days, the $\sigma$ of the $\mathrm{O}-\mathrm{C}$ residuals drops to $32.9 \mathrm{~ms}^{-1}$, with a $\chi^{2}$ of 0.60 , and no strong power remains in the Scargle plot.

\subsection{Line bisector and activity analysis}

Radial velocity measurements of giant stars may be significantly affected by stellar surface phenomena not related to the presence of an orbiting planet. Short-period, from hours to days, and long-term, of a hundred of days, radial velocity variations have been known to exist in giant stars for many years (e.g. Hatzes \& Cochran 1993, 1994). The short-period variations are, very 


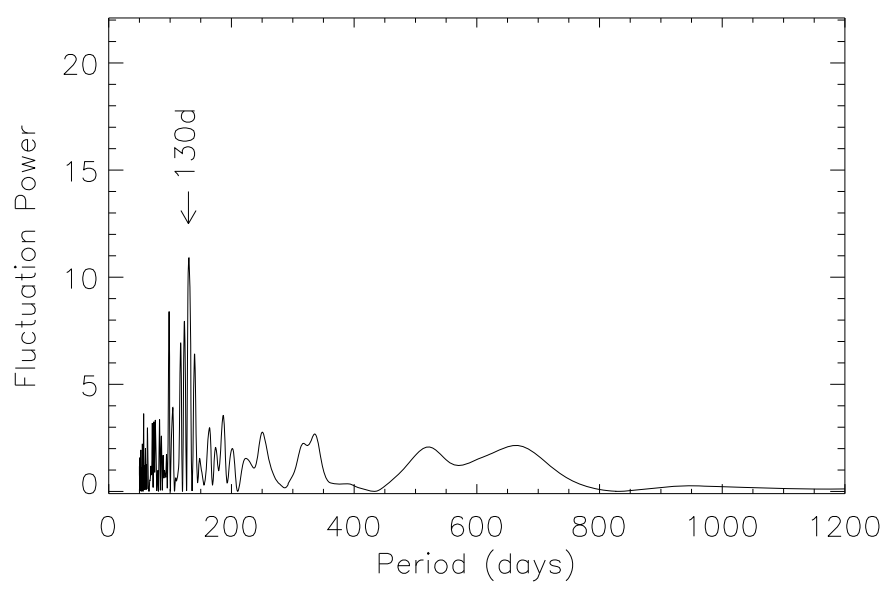

Fig. 5. The Lomb Scargle periodogram of the radial velocity residuals, with a significant power at a period of 130 days.

probably, the result of stellar pulsations (e.g.: Frandsen et al. 2002; Hekker et al. 2006). The long-term variations are explained by three main phenomena: the presence of an orbiting companion, rotational modulation of surface features and longperiod, non-radial oscillation modes. The basic tools to study the origin of radial velocity variations derived from stellar spectra are the analysis of shapes of the spectral lines using the bisector velocity span (Queloz et al. 2001; Hatzes 1996) and of the $\mathrm{CaII} \mathrm{H} \& \mathrm{~K}$ emission lines. The bisector velocity span traces line shape variations and should remain constant if the variability of the measured radial velocities is due to an orbiting companion. Such a procedure has also been successfully applied to detect rotational modulation (Setiawan et al. 2003, 2004, 2005).

We have computed the bisector velocity span for HD 110014, displayed in Fig. 6 as a function of the measured radial velocity. The bisector span shows no correlation with radial velocity variations. A statistical analyses of the radial velocity and bisector velocity span measurements gives a correlation coefficient of 0.48 , with a probability of 0.0015 that they are correlated.

Figure 7 shows the spectrum of HD 110014 in the CaII H and $\mathrm{K}$ region. The core of these deep lines is formed in the chromosphere and shows a typical central reversal in the presence of chromospheric activity (e.g.: Pasquini et al. 1988). The central reversal in HD 110014 is present, but very small, indicating a low level of chromospheric activity for this star, corroborated by the CaII $\mathrm{K}$ absolute surface flux, $\log (\mathrm{FK})=4.97$, typical of inactive giant stars (Pasquini et al. 2000). In addition, we searched for variability in the CaII $\mathrm{K}$ emission core, by computing the emission line intensity relative to the intensities of regions in the blue and red wings close to the core, which contains no strong absorption features. Figure 8 shows the relative $\mathrm{CaII} \mathrm{K}$ indices as a function of the radial velocity measurements, as well as a Lomb-Scargle periodogram of the referred indices. Indeed, this analysis shows no correlation between the computed indices and the radial velocity, and, in particular, no significant power is seen around 130 days, namely the periodicity resulting from the residuals of radial velocity.

\subsection{Hipparcos photometry}

HD 110014 is listed by Aldeman (2001) as one of the least variable stars from the Hipparcos survey, with a photometric variability down to $0.01 \mathrm{mag}$, without any sign of systematic variability. The Hipparcos mean magnitude for this star is

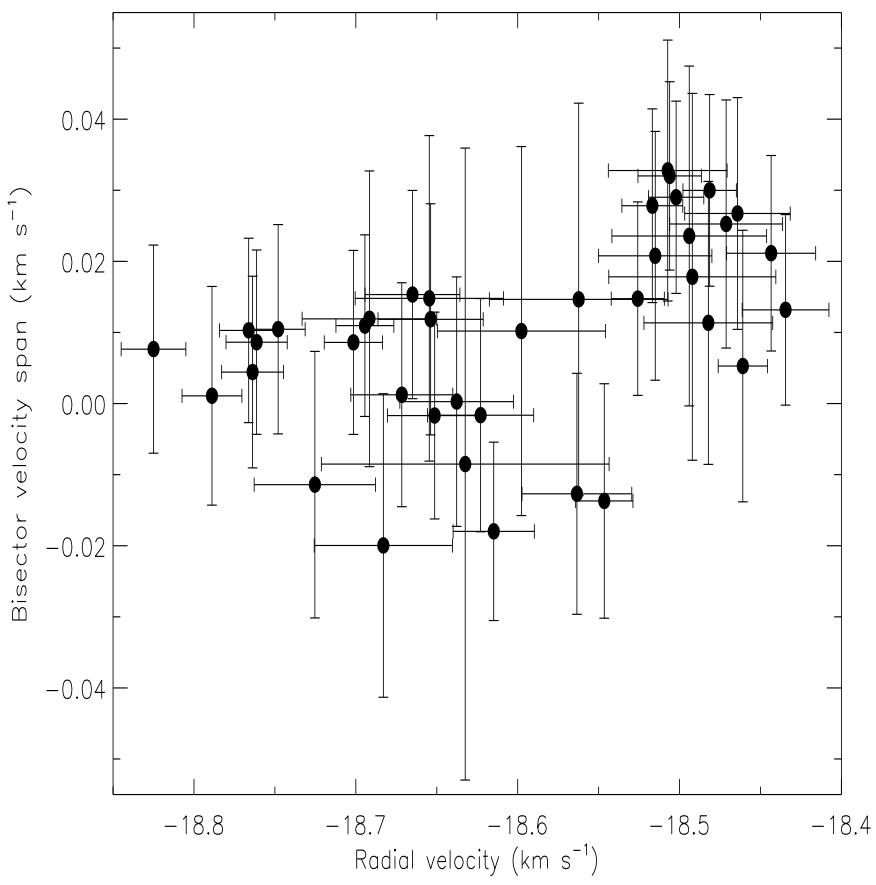

Fig. 6. The bisector velocity span of HD 110014, calculated from the spectra obtained in the present survey. The diagram shows no correlation between bisector velocity spans and the measured radial velocities.
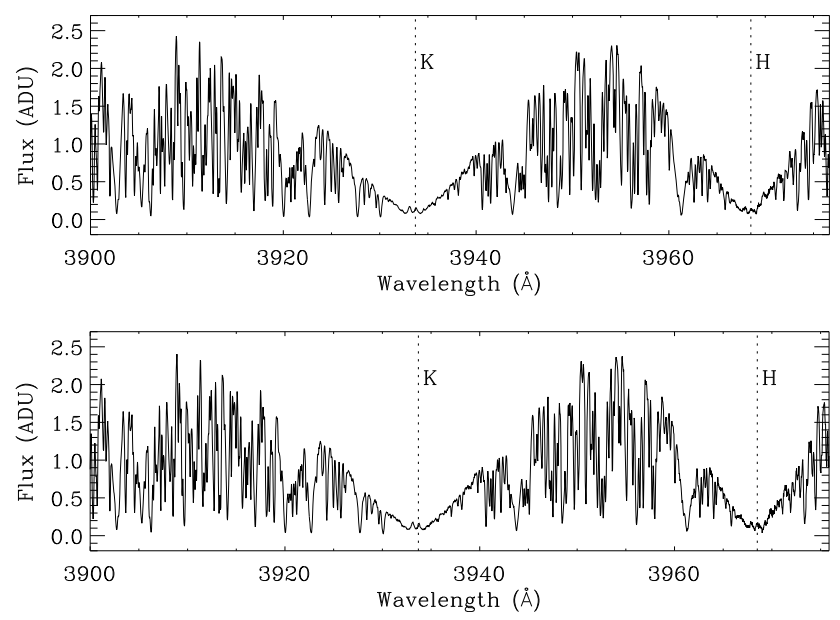

Fig. 7. The CaII H \& K spectral region for HD 110014. No re-emission features are visible in the line cores, indicating a low level of activity. Upper panel for the lower part of RV curve and lower panel for the upper part of RV curve.

$4.8251 \pm 0.0007 \mathrm{mag}$. Despite this solid constraint, we have performed a deeper search for any possible periodicities in the Hipparcos photometric data of HD 110014 , by computing a Lomb-Scargle periodogram on the referred photometric data which amounts to 89 measurements. From such an analysis, in particular, no excess fluctuation power is found at and around the 835-day period detected in the radial velocity measurements. In addition, no real peak is found around the 130-day period. The Lomb-Scargle periodogram of the Hipparcos photometric data for HD 110014 is displayed in Fig. 9. 

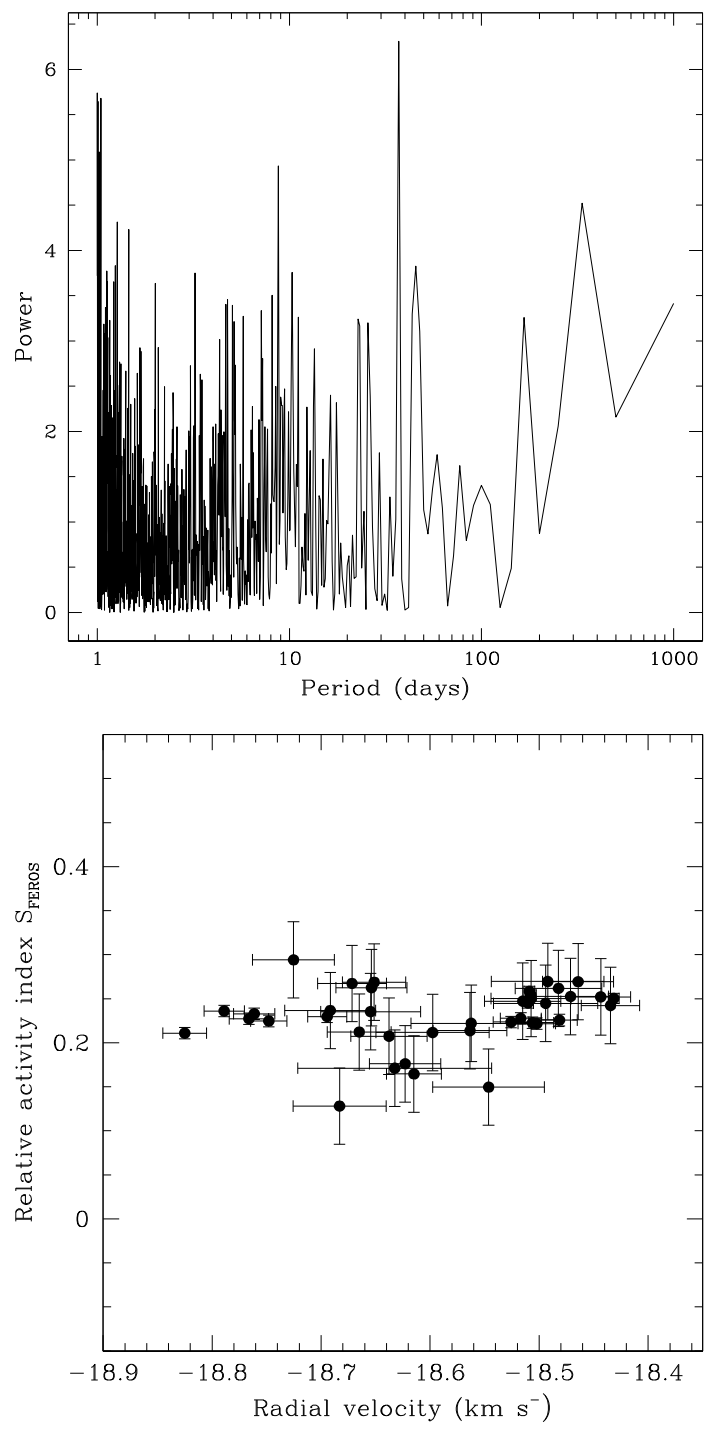

Fig. 8. The relative CaII $\mathrm{K}$ indices as a function of radial velocity measurements (lower panel). A Lomb-Scargle periodogram of the relative $\mathrm{CaII} \mathrm{K}$ indices (upper panel).

\section{Results and discussion}

This work presents a compelling evidence for a planetary mass companion orbiting HD 110014, a K2III intermediate-mass giant star. A strictly periodic variability in radial velocity is detected on the basis of our observations, from which we computed a period of $835.477 \pm 6.04$ days. From a Keplerian orbit solution, this period indicates for the detected extrasolar planet a minimum mass $M \sin i=9.5 M_{\mathrm{Jup}}$ in a middle eccentric orbit, $e=0.462 \pm 0.069,2.1 \mathrm{AU}$ away of the central star. We have examined the spectral line shapes, the CaII emission and Hipparcos photometry, to search for other forms of variability that could play a role on the radial velocity variation. No dependence between the asymmetry of the spectral line profile and the measured radial velocities was observed. Again, no significant chromospheric activity is observed in the $\mathrm{CaII} H \& \mathrm{~K}$ core region, corroborated by a CaII K absolute surface flux, $\log (\mathrm{FK})=4.97$, typical of inactive giant stars. It is unlikely also that the 835-day radial velocity variation of the radial velocity of HD 110014 is due to rotational modulation. The upper limit of the rotation period, $P_{\text {rot }} / \sin i \sim 530$ days, is clearly much lower than the such a

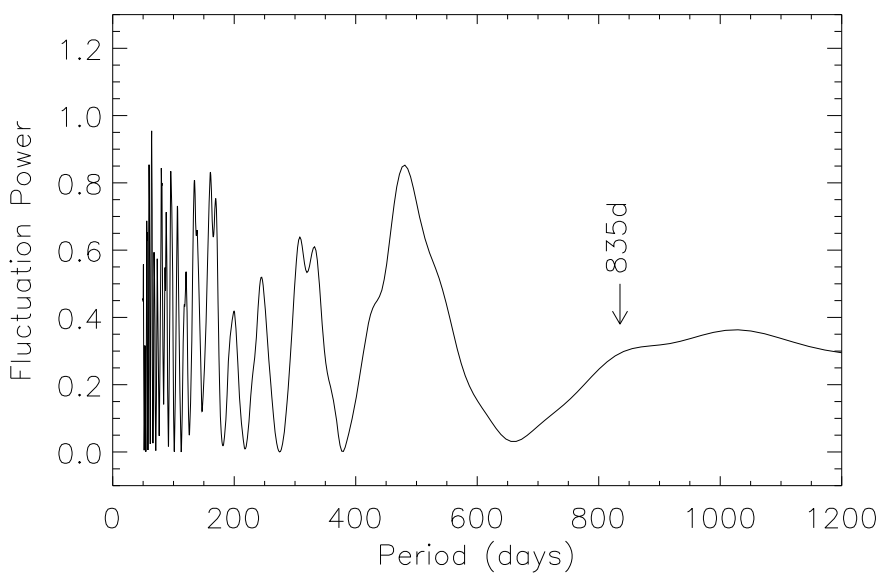

Fig. 9. A periodogram of the HIPPARCOS photometric measurements of HD 110014. The vertical arrow marks the corresponding 835-day period, for reference, close to the observed period in the radial velocity variation.

period. Finally, the Hipparcos photometry data does not present any significant variation at the orbital period of 835 days.

The root-cause of the 130-day periodicity is no yet clear. In spite of the fact that Hipparcos photometry measurements were not contemporaneous with the present radial velocity survey for HD 110014, there is no clear similar feature in the photometric data analysis. In this context, it appears that there is no connection between such a periodicity and stellar activity. One can also inquire on a possible link between the 130-day periodicity and the stellar rotation. For instance, with the obtained upper limit for the rotation period, $P_{\text {rot }} / \sin i \sim 530$ days, and an angle $i$ lower than about 15 degrees one can reproduce easily the 130-day period. We note that at such a small inclination angle the spot features responsible for the RV variations would have to be situated near the rotation pole of the star. Since such features would always be in view such spots would produce an RV curve that is more sine-like, not the "sawtooth" pattern actually observed for HD 110014 that is easily explained by eccentric Keplerian motion. Finally, could this variability possibly be due to a second planetary companion? Let us recall that Sato et al. (2008) have detected 2 short-period planetary companions, with 136.7 days and 184.02 days, orbiting, respectively, the intermediate-mass giant stars $\xi$ Aquilae (K0III) and HD 81688 (K0III-IV), and Sato et al. (2003) claimed for a planetary companion with a 199.5-day period around HD 104985(G9III). A second planet orbiting HD 110014, with 130-day orbital period, it would represent a very interesting step towards our understanding of planet surviving around evolved stars, in particular because HD 110014 can be at the end of its core helium burning phase (although, as we saw above, the more massive solution, ascending the RGB is favored by the high ${ }^{12} \mathrm{C} /{ }^{13} \mathrm{C}$ ratio). In addition, it is interesting to underline that the period of 130 days is close to a $6: 1$ resonance of the 835 -day period. Clearly, additional RV measurements are required for convincing argument favouring a second planetary companion around this star. To this purpose, we continue to monitor HD 110014, carrying out additional analysis of other observable, including $\mathrm{H} \alpha$ line variability and other spectral indicators (like CNO lines) to better constrain on observational basis its evolutionary status.

Acknowledgements. Research activities of the Stellar Board at the Federal University of Rio Grande do Norte are supported by continuous grants from CNPq and FAPERN Brazilian Agencies. We thank Miss Sânzia Alves for assistance in the preparation of the art of the present paper. This research has made use of data provided by SIMBAD data basis, operated by CDS, Strasbourg, France. 


\section{References}

Aldeman, S. J. 2001, A\&A, 367, 297

Baranne, L., Queloz, D., Mayor, M., et al. 1996, A\&AS, 119, 373 Benz, W., \& Mayor, M. 1984, A\&A

Biazzo, K., Pasquini, L., Girardi, L., et al. 2007, A\&A 475, 981

da Silva, L., Girardi, L., Pasquini, L., et al. 2006, A\&A, 458, 609 de Medeiros, J. R., \& Mayor, M. 1999, A\&AS, 139, 433

de Medeiros, J. R., Da Rocha, C., \& Mayor, M. 1996, A\&A, 314, 499

Döellinger, M. P., Hatzes, A. P., Pasquini, L., et al. 2007, A\&A, 472, 649

Duncan, M. J., \& Lissauer, J. J. 1998, Icarus, 134, 303

ESA 1997, The Hipparcos and Tycho Catalogues, ESA SP-1200

Flower, P. J. 1996, ApJ, 469, 365

Frandsen, S., Carrier, F., Aerts, C., et al. 2002, A\&A, 394, L5

Frink, S., Mitchell, D. S., Quirrenbach, A., et al. 2002, ApJ, 576, 478

Giradi, L., Bertelli, G., Bressan, A., et al. 2002, A\&A, 391, 195

Hekker, A., Aerts, C., de Ridder, J., \& Carrier, F. 2006, A\&A, 458, 931

Hatzes, A. 1996, PASP, 108, 839

Hatzes, A., \& Cochran, W. D. 1993, ApJ, 413, 339

Hatzes, A., \& Cochran, W. D. 1994, ApJ, 422, 366

Hatzes, A., \& Cochran, W. D., Endl, M., et al. 2003, ApJ, 599, 1383

Hatzes, A., Guenther, E. W., Endl, M., et al. 2005, A\&A, 437, 743

Johnson, J. A., Fischer, D. A., Marcy, G. W., et al. 2007, ApJ, 665, 785

Jørgensen, B. R., \& Lindegren, L. 2005, A\&A, 436, 127
Lejeune, T., Cuisinier, F., \& Buser, R. 1998, A\&AS, 130, 65

Lovis, C., \& Mayor, M. 2007, A\&A, 472, 657

Mayor, M., \& Queloz, D. 1995, Nature, 378, 355

Melo, C., Pasquini, L., \& de Medeiros, J. R. 2001, A\&A, 375, 851

Naef, D., Mayor, M., Benz, W., et al. 2007, A\&A, 470, 721

Niedzielski, A., Konacki, M., et al. 2007, ApJ, 669, 1354

Pasquini, L., Pallavicini, R., \& Pakull, M. 1988, A\&A, 191, 253

Pasquini, L., de Medeiros, J. R., \& Girardi, L. 2000, A\&A, 361, 1011

Pasquini, L., Dollinger, M. P., Weiss, A., et al. 2007, A\&A, 473, 979

Queloz, D., Henry, W., Sivan, J. P., et al. 2001, A\&A, 379, 279

Reffert, S., Quirrenbach, A., Mitchell, D. L., et al. 2006, ApJ, 652, 661

Sato, B., Ando, H., Kambe, E., et al. 2003, ApJ, 597, L157

Sato, B., Izumiura, H., Toyota, E., et al. 2007, ApJ, 661, 527

Sato, B., Izumiura, H., Toyota, E., et al. 2008, PASJ, 60, 539

Setiawan, J., Hatzes, A., von der Luhe, O., et al. 2003, A\&A, 398, L19

Setiawan, J., Pasquini, L., da Silva, L., et al. 2004, A\&A, 421, 241

Setiawan, J., Rodmann, J., da Silva, L., et al. 2005, A\&A, 437, 31

Scargle, J. D. 1982, ApJ, 263, 835

Smiljanic, R., Gauderon, R., et al. 2008, A\&A, in press [arXiv:0810.1701v1]

Tomkin, J. , Luck, R. E., \& Lambert, D. L. 1976, ApJ, 210, 694

van Leeuwen, F. 2007, Hipparcos, the new reduction of the raw data (Dordrecht: Springer)

van Leeuwen, F. 2008, A\&A, 474, 653

van Leeuwen, F., \& Fantino, E. 2005, A\&A, 439, 791 